\title{
Sparse Multiple Relay Selection for network beamforming with Individual Power Constraints Using Semidefinite Relaxation
}

\author{
Luis Blanco and Montse Nájar, Senior Member, IEEE
}

\begin{abstract}
This paper deals with the multiple relay selection problem in two-hop wireless cooperative networks with individual power constraints at the relays. In particular, it addresses the problem of selecting the best subset of $K$ cooperative nodes and their corresponding beamforming weights so that the signal-to-noise ratio (SNR) is maximized at the destination. This problem is computationally demanding and requires an exhaustive search over all the possible combinations. In order to reduce the complexity, a new sub-optimal method is proposed. This technique exhibits a near-optimal performance with a computational burden that is far less than the one needed in the combinatorial search. The proposed method is based on the use of the $l_{1}$-norm squared and the Charnes-Cooper transformation and naturally leads to a semidefinite programming relaxation with an affordable computational cost. Contrary to other approaches in the literature, the technique exposed herein is based on the knowledge of the second-order statistics of the channels and the relays are not limited to cooperate with full power.
\end{abstract}

Index Terms-Wireless relay network, multiple relay selection, distributed beamforming, sparsity-promoting norms, semidefinite relaxation, combinatorial optimization.

\section{INTRODUCTION}

As it is well-known, relay systems increase the spatial diversity and the reliability of wireless communications systems. The simplest relay network consists of a single source and $N$ relays that cooperate to send to a destination node the message transmitted by the source. Relay communications systems can be classified taking into account how the relays process the information received from the source node. The most popular cooperative schemes available in the wireless relay literature are: amplify-and-forward (AF), decode-and-forward [1], compressand-forward and coded-cooperation [2]. Nonetheless, amplifyand-forward has attracted special interest due to its simplicity. In this context, distributed relay beamforming, also known as cooperative beamforming, has been shown to be a powerful technique which provides power efficiency and is able to increase the communications reliability. In general terms, in distributed beamforming the relays cooperate acting as virtual

The research leading to these results has received funding from the European Commission in the framework of the FP7 Network of Excellence in Wireless COMmunications NEWCOM\# (Grant agreement no.318306) and from the Catalan Government under grants 2014-SGR-60 and 2014-SGR1551.

L. Blanco is with the Telecommunications Technological Centre of Catalonia (CTTC), 08860 Castelldefels, Barcelona, Spain (e-mail:lblanco@cttc.es).

M. Nájar is with the Department of Signal Theory and Communications, Technical University of Catalonia (UPC), 08034 Barcelona, Spain (email:montse.najar@upc.edu). antenna and adjust their transmission weights to form a beam to the destination. Since each relay multiplies its received signal by a complex weight and retransmits it, the beamforming weights have to be determined according to some optimality criterion. Different beamforming approaches have been considered in the literature [3]. One such criteria is the minimization of the total transmitted power subject to a given constraint on the quality of service at the receiver. The second approach is the maximization of the received SNR subject to certain power constraints, i.e., individual power constraints in each relay or on the total power transmitted by the relays. Due to the fact that relay nodes have particular constraints on the battery lifetimes, individual power constraints in each relay are of practical interest. It is worth noting that the optimal solution of this maximization problem results in relay powers which do not correspond, in general, to their maximum allowable values, i.e., to achieve the maximum SNR at the receiver, the relays may not use their maximum allowable power.

In the conventional cooperation strategies exposed above, all the relays cooperate in relaying the signals. This is the optimal strategy from the point of view of the end-to-end performance. Nevertheless, in practical scenarios, the benefits of the cooperation could be offset by the cost of the cooperation and the consumption of additional system resources. In many cases, it is impractical or even unfeasible to activate all (or many) relays (see the references [4]-[9]). For instance, in many practical situations the number of potential cooperating nodes could be large, e.g., in device-to-device communication networks or in wireless sensor networks, and the benefits of the cooperation with all the relays can be outweighed by the costs of the cooperation. These costs include the signaling overhead and the efforts needed to maintain the synchronization between all the nodes [4]-[7]. In this context, by considering relay selection, the overall processing in the network can be simplified achieving a significant reduction in the implementation complexity [4], [6]-[9].

There exist a vast literature devoted to relay selection. Most of these schemes are based on single relay selection, i.e., only one of the relay nodes can be selected to cooperate in the retransmission, e.g. [10] and [11]. Nevertheless, in adverse environments, transmitting over a single relay may not be sufficient to achieve the desired performance at the destination. This has motivated the generalization of this idea, allowing more than one node to cooperate. Multiple relay selection for a single source-destination pair has attracted attention in some references [9], [12]-[14]. In all these approaches, for 
simplicity, the relays are not allowed to adjust their transmit power arbitrarily, i.e., each relay has only two choices: to cooperate with full power or not to cooperate at all. In [9], the authors propose several SNR-suboptimal multiple relay selection techniques based on some ordering functions. In [13], Laneman et al. proposed a Decode-and-Forward protocol that allow the relays to cooperate if the channel between the source and the relays, the so-called backward channel, exceeds a fixed threshold. On the contrary, the scheme proposed in [12] is based on the full Channel State Information (CSI) of the backward and the forward channels of each relay. Therein two different problems have been tackled: the minimization of the end-to-end error performance under a total power constraint, and the dual problem, the minimization of the total power consumption constrained to a maximum error probability. These problems fall within the class of the socalled 0-1 Knapsack problems and are solved by taking use of several greedy algorithms. A similar approach was proposed for cognitive relay networks in [14].

This paper deals with problem of multiple relay selection for distributed beamforming under individual power constraints at the relay nodes. In particular, it addresses the problem of finding the best subset of cooperative nodes, and their beamformer weights, so that the SNR is maximized at the destination. The selection of the best subset of $K$ nodes out of a set of $N$ potential relays with individual relay power constraints is a NP-hard problem which requires an exhaustive search over all the possible sparsity patterns. In order to reduce the computational burden, this paper proposes a sub-optimal method which exhibits a performance which is very close to the SNR-optimal multiple relay scheme with a reduced complexity. The proposed method is based on the knowledge of the second-order statistics of the CSI and in contrast to other approaches in the relay selection literature [9], [12]-[14], in the technique proposed herein, the relays are not limited to cooperate only with full power. Interestingly enough, this optimization leads to results in which the powers of the selected set of relays do not correspond to the maximum allowable values. The proposed algorithm is based on the Charnes-Cooper transformation and the $l_{1}$-norm squared [15], a surrogate of the $l_{0}$-norm which enforces zeros in the relay powers, and naturally yields a semidefinite programming problem (SDP).

It is important to remark that the sparse multiple relay selection problem described in this paper does not belong to the class of the so-called sparse recovery problems in which a solution vector with few non-zero elements has to be estimated and the conditions that ensure the exact recovery of the true sparsity pattern need to be studied. The adjective sparse herein relates to the fact that the algorithm proposed for the selection of the relays uses a sparsity-inducing norm, the $l_{1}$-norm squared, that promotes the appearance of zeros in the final solution and consequently performs the subset selection.

The paper is organized as follows. Section II describes the signal model and presents the multiple relay selection problem. The algorithm proposed for the selection of the cooperating nodes is derived in Section III and the analysis of its performance is shown in Section IV. Finally, some

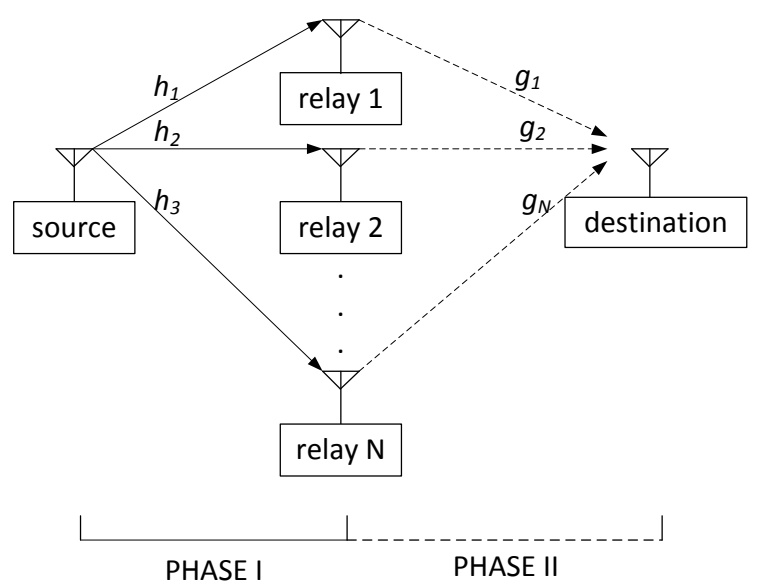

Fig. 1: Wireless relay network

concluding remarks are provided in Section V.

Notation: In the following, matrices and vectors are denoted by boldface uppercase letters and lower case letters, respectively; For a given matrix $\mathbf{A}, \mathbf{A}^{H}, \operatorname{Tr}\{\mathbf{A}\}, \operatorname{rank}(\mathbf{A}),|\mathbf{A}|$, $\|\mathbf{A}\|_{2}^{2}$ denote the conjugate transpose, the trace, the rank, the element-wise absolute value and the square of the Frobenius norm, respectively. $A_{i j}$ stands for the element in the $i$ th row and $j$ th column of $\mathbf{A}$. The $k$ th element of a vector $\mathbf{x}$ is denoted by $x_{k}$. The Euclidean norm of this vector is $\|\mathbf{x}\|_{2}$ and $\|\mathbf{x}\|_{1}$ is the $l_{1}$-norm. Finally, for a set $S,|S|$ denotes its cardinality and $S(i)$ stands for the $i$ th element of the set.

\section{SYSTEM MODEL AND PROBLEM FORMULATION}

Consider a two-hop wireless cooperative network which consists of a source, a destination and $N$ potential relays as it is shown in Fig. 1. Each of the nodes of this scheme is equipped with a single antenna. For sake of simplicity it is assumed that due to the poor quality of the channel between the source and the destination, there is no direct link between them. The channel between the source and the $i$ th relay and the channel between the $i$ th relay and the destination are denoted by $h_{i}$ and $g_{i}$, respectively.

Even though, the pioneering studies on network relay beamforming have assumed that the instantaneous CSI is perfectly known at the relays or at the destination node [16]. Unfortunately, this assumption is often violated in practical scenarios. To avoid the need to know the instantaneous CSI, the flat fading channel coefficients $\left\{h_{i}\right\}_{i=1}^{N}$ and $\left\{g_{i}\right\}_{i=1}^{N}$ can be modeled as random values. Similar to [3], [17], [18] and [19], in this paper it is assumed that the joint second-order statistics of these channels are known at a central node, for instance, at the destination node, which is the one in charge of computing the relay weights taking into account past observations, and then distributing them to the relay nodes via a dedicated channel. This assumption allows us to consider some uncertainty in the channel models through introducing the covariance matrices of the channel gains. Since the coefficients of the channels are relatively stable in stationary environments 
and can be estimated using past observations, it is reasonable to assume the availability of the second order statistics of the channels.

In the scheme presented herein, we have considered a twostep amplify-and-forward protocol for the communication between the source and the destination. During the first step (slot) the source broadcasts the signal $\sqrt{P_{s}} s$ to the relays, where $P_{s}$ denotes the transmit power and $s$ is the information symbol. Without loss of generality it is assumed that $E\left\{|s|^{2}\right\}=1$. The signal received at the $i$ th relay is given by

$$
x_{i}=\sqrt{P_{s}} h_{i} s+\eta_{i},
$$

where $\eta_{i}$ denotes the additive noise at the $i$ th relay whose variance is known to be $\sigma_{r}^{2}$. In the second step the $i$ th relay transmits a weighted version of its received signal. This can be expressed as

$$
y_{i}=w_{i} x_{i} .
$$

The received signal at the destination node is given by

$$
r=\sqrt{P_{s}} \sum_{i=1}^{N} w_{i} h_{i} g_{i} s+\sum_{i=1}^{N} w_{i} g_{i} \eta_{i}+n_{d},
$$

where $n_{d}$ is the noise at the destination which has a known variance $\sigma_{d}^{2}$. Note that whereas the first term in (3) corresponds to the desired signal component, the sum of the second and the third term is the total noise received at the destination.

\section{A. SNR maximization with individual power constraints with- out relay selection}

The aim of this subsection is to briefly describe the classical design problem presented in [3]. The maximization of the SNR at the destination under transmit power constraints at each relay is given by

$$
\max _{\mathbf{w}} \operatorname{SNR} \quad \text { s.t. } \quad p_{i} \leq P_{i} \quad \forall i=1, \ldots, N,
$$

where $\mathbf{w}=\left[w_{1} \ldots w_{N}\right]^{T}$ is the network beamforming vector and $p_{i}$ and $P_{i}$ are the actual transmit power and the maximum allowable transmit power of the $i$ th relay, respectively. Note that in the problem exposed above relay selection is not considered.

The expected power of the desired signal component in the expression (3), that is, the expected power of the first term in the sum is given by

$$
P_{d}=E\left\{\left|\sqrt{P_{s}} \sum_{i=1}^{N} w_{i} h_{i} g_{i} s\right|^{2}\right\}=\mathbf{w}^{H} \mathbf{A} \mathbf{w},
$$

where $\mathbf{A}=P_{s} E\left\{(\mathbf{h} \odot \mathbf{g})(\mathbf{h} \odot \mathbf{g})^{H}\right\}$. In the latter expression, the operator $\odot$ represents the Schur-Hadamard product and $\mathbf{h}=\left[h_{1} \ldots h_{N}\right]^{T}, \mathbf{g}=\left[g_{1} \ldots g_{N}\right]^{T}$. This matrix can be decomposed as a diagonal matrix plus a rank-one matrix [18] (DPR1). Assuming that the coefficients of the backward and the forward channels, $\left\{h_{i}\right\}_{i=1}^{N}$ and $\left\{g_{i}\right\}_{i=1}^{N}$, are statistically independent, the $(i, j)$ th element of $\mathbf{A}$, denoted by $A_{i, j}$, can be expressed as follows

$$
A_{i j}= \begin{cases}P_{s} E\left\{\left|h_{i}\right|^{2}\right\} E\left\{\left|g_{i}\right|^{2}\right\} & \text { if } i=j \\ P_{s} E\left\{h_{i}\right\} E\left\{g_{i}\right\} E\left\{h_{j}^{*}\right\} E\left\{g_{j}^{*}\right\} & \forall i \neq j .\end{cases}
$$

Let $\bar{h}_{i}=E\left\{h_{i}\right\}, \quad \bar{g}_{i}=E\left\{g_{i}\right\}, \alpha_{i}=E\left\{\left|h_{i}-\bar{h}_{i}\right|^{2}\right\}$ and $\beta_{i}=E\left\{\left|g_{i}-\bar{g}_{i}\right|^{2}\right\}$. It is straightforward to rewrite $\mathbf{A}$ as

$$
\mathbf{A}=\boldsymbol{\Lambda}+\mathbf{v} \mathbf{v}^{H},
$$

with $\boldsymbol{\Lambda}=P_{s} \operatorname{diag}\left(\beta_{1}\left|\bar{h}_{1}\right|^{2}+\alpha_{1}\left|\bar{g}_{1}\right|^{2}+\alpha_{1} \beta_{1}, \ldots, \beta_{N}\left|\bar{h}_{N}\right|^{2}+\right.$ $\left.\alpha_{N}\left|\bar{g}_{N}\right|^{2}+\alpha_{N} \beta_{N}\right)$ and $\mathbf{v}=\sqrt{P_{s}}\left[\bar{h}_{1} \bar{g}_{1}, \ldots, \bar{h}_{N} \bar{g}_{N}\right]^{T}$.

The total noise power, denoted as $P_{n}$, is defined as

$$
P_{n}=E\left\{\left(\sum_{i=1}^{N} w_{i} g_{i} \eta_{i}+n_{d}\right)\left(\sum_{j=1}^{N} w_{j} g_{j} \eta_{j}+n_{d}\right)^{*}\right\} .
$$

Assuming that $\eta_{i}$ is a zero-mean additive noise and that $\left\{\eta_{i}\right\}_{i=1}^{N}$ and $n_{d}$ are mutually independent random variables, then the noise power can be formulated as

$$
P_{n}=\mathbf{w}^{H} \mathbf{B w}+\sigma_{d}^{2}
$$

where

$$
\mathbf{B}=\sigma_{r}^{2} \operatorname{diag}\left(E\left\{\left|g_{1}\right|^{2}\right\}, E\left\{\left|g_{2}\right|^{2}\right\}, \ldots, E\left\{\left|g_{N}\right|^{2}\right\}\right) .
$$

Hence, the SNR at destination node is defined as

$$
\mathrm{SNR}=\frac{P_{d}}{P_{n}}=\frac{\mathbf{w}^{H} \mathbf{A} \mathbf{w}}{\mathbf{w}^{H} \mathbf{B w}+\sigma_{d}^{2}} .
$$

In order to specify the power constraints at the relays in (4), the average transmit power of the $i$ th relay, denoted as $p_{i}$, is defined as follows

$$
p_{i}=E\left\{\left|x_{i}\right|^{2}\right\}\left|w_{i}\right|^{2}=D_{i i}\left|w_{i}\right|^{2},
$$

where $D_{i i}$ is the $i$ th element of diagonal of the matrix $\mathbf{D}$ given by

$$
\mathbf{D}=P_{s} \operatorname{diag}\left(E\left\{\left|h_{1}\right|^{2}\right\}, \ldots, E\left\{\left|h_{N}\right|^{2}\right\}\right)+\sigma_{r}^{2} \mathbf{I} .
$$

The maximization of the SNR under individual relay power constraints exposed in (4) can be formally expressed as

$$
\begin{array}{ll}
\max _{\mathbf{w}} & \frac{\mathbf{w}^{H} \mathbf{A} \mathbf{w}}{\mathbf{w}^{H} \mathbf{B w}+\sigma_{d}^{2}} \\
\text { s.t. } & D_{i i}\left|w_{i}\right|^{2} \leq P_{i} \quad \forall i=1, \ldots, N .
\end{array}
$$

Note that since $\mathbf{B}$ is diagonal and $\mathbf{A}$ is a diagonal plus rankone matrix, the phases of the optimal beamformer only depend on the entries of the vector $\mathbf{v}$ defined in (7). In particular, the phases of the optimal weights can be obtained as $\angle w_{i}=\angle v_{i}$, where $v_{i}$ denotes the $i$ th element of $\mathbf{v}$.

Considering $\mathbf{X}=\mathbf{w} \mathbf{w}^{H}$, the problem (14) can be rewritten as

$$
\begin{array}{cl}
\max _{\mathbf{X}} & \frac{\operatorname{Tr}\{\mathbf{A} \mathbf{X}\}}{\operatorname{Tr}\{\mathbf{B X}\}+\sigma_{d}^{2}} \\
\text { s.t. } & X_{i i} \leq P_{i} / D_{i i} \quad \forall i=1, \ldots, N \\
& \mathbf{X} \succeq 0 ; \operatorname{rank}(\mathbf{X})=1,
\end{array}
$$


where $X_{i i}$ denotes the $i$ th diagonal element of $\mathbf{X}$. Following the idea of semidefinite relaxation and dropping the nonconvex rank constraint, the problem presented above can be relaxed as follows

$$
\begin{array}{ll}
\max _{\mathbf{X}, t} & t \\
\text { s.t. } & \operatorname{Tr}\{\mathbf{X}(\mathbf{A}-t \mathbf{B})\} \geq \sigma_{d}^{2} t \\
& X_{i i} \leq P_{i} / D_{i i} \quad \forall i=1, \ldots, N ; \quad \mathbf{X} \succeq 0 .
\end{array}
$$

The latter problem is quasiconvex and the standard approach is to solve it by means of a bisection search method in which the optimal solution is obtained iteratively after solving a sequence of (often many) semidefinite programming problems (for further information see reference [3]).

\section{B. Multiple relay selection for SNR optimization with individ- ual constraints}

Let us consider the joint problem of selecting the best subset of $K$ nodes out of the set of $N$ potential relays and the estimation of the weights which maximize the SNR at the destination, subject to individual power constraints at the relays. Mathematically, this problem can be expressed as

$$
\begin{array}{cl}
\max _{\mathbf{w}} & \frac{\mathbf{w}^{H} \mathbf{A} \mathbf{w}}{\mathbf{w}^{H} \mathbf{B w}+\sigma_{d}^{2}} \\
\text { s.t. } & D_{i i}\left|w_{i}\right|^{2} \leq P_{i} \quad \forall i=1, \ldots, N \\
& \operatorname{card}(\mathbf{w})=K,
\end{array}
$$

where $K>0$ is a given constant and $\operatorname{card}(\mathbf{w})=K$ is the number of non-zero coefficients in the vector $w$. It is worth mentioning that adding zeros in the network beamforming vector is equivalent to selecting the best subset of relays. Note that the $k$-th relay is excluded from the transmission if the $k$-th component of the solution vector $\mathbf{w}$ is equal to zero. The problem in (17) is a NP-hard problem and requires an exhaustive search over all the possible $\left(\begin{array}{c}N \\ K\end{array}\right)$ sparsity patterns. This search is computationally unaffordable and this fact motivates the pursuit of an efficient algorithm with a nearoptimal performance.

\section{THE PROPOSED METHOD}

\section{A. Selection of the subset of relays}

The problem considered in (17) is non-convex and the aim of this subsection is to derive a convex relaxation of this problem in order to obtain a new algorithm with a lower computational complexity. The traditional way of deriving convex approximations of cardinality-constrained problems in combinatorial optimization is to replace the cardinality operator by the $l_{1}$-norm [20], [21], defined as $\|\mathbf{w}\|_{1}=\sum_{i=1}^{i=N}\left|w_{i}\right|$. As it is well-known, the $l_{1}$-norm is the tightest convex relaxation of the cardinality operator [21] and has a sparsifying effect that has long been observed in statistics and signal processing [22]. Nonetheless, a different approach is considered in this paper. Similar to [15] and [23], the $l_{1}$-norm squared, denoted as $\|\mathbf{w}\|_{1}^{2}$, is considered instead of the traditional $l_{1}$-norm. The rationale behind the use of the $l_{1}$-norm squared as a surrogate of the cardinality is twofold. First, it is a sparsity-inducing norm which encourages the appearance of null components in the network beamforming vector and, consequently, performs the subset selection. Second, the problem that results after considering the $l_{1}$-norm squared naturally yields a semidefinite programming relaxation, something that is not obvious when the $l_{1}$-norm is considered instead. Therefore, let us relax the problem presented in (17) rewriting it in terms of the $l_{1}$-norm squared

$$
\begin{array}{cl}
\max _{\mathbf{w}} & \frac{\mathbf{w}^{H} \mathbf{A} \mathbf{w}}{\mathbf{w}^{H} \mathbf{B w}+\sigma_{d}^{2}} \\
\text { s.t. } & D_{i i}\left|w_{i}\right|^{2} \leq P_{i} \quad \forall i=1, \ldots, N \\
& \|\mathbf{w}\|_{1}^{2} \leq \gamma,
\end{array}
$$

where $\gamma$ is a positive parameter that controls the sparsity of the beamforming vector, i.e., the number of active components in $\mathbf{w}$. Let us skip now the discussion about how to adjust the parameter $\gamma$ to properly perform the subset selection. Later, in the following subsection, this problem will be addressed and it will be explained how to adjust the parameter $\gamma$ to obtain a solution of (18) with only $K$ active entries.

Unfortunately, the problem in (18) is still NP-hard and this motivates the use of a semidefinite relaxation to handle it. First, let us define $\mathbf{X} \triangleq \mathbf{w} \mathbf{w}^{H} \in H_{N}^{+}$, i.e., a $N \times N$ Hermitian positive semidefinite matrix. Then, the constraint (18c) can be rewritten as

$$
\|\mathbf{w}\|_{1}^{2}=\left(\sum_{i=1}^{N}\left|w_{i}\right|\right)^{2}=\mathbf{1}_{N}^{T}|\mathbf{X}| \mathbf{1}_{N}
$$

where $|\mathbf{X}|$ denotes the element-wise absolute value of the matrix $\mathbf{X}$ and $\mathbf{1}_{N}$ the all-one column vector of length $N$. By substituting the $l_{1}$-norm squared by its equivalent formulation (19), the problem in (18) can be expressed as

$$
\begin{array}{cl}
\max _{\mathbf{X}} & \frac{\operatorname{Tr}\{\mathbf{A X}\}}{\operatorname{Tr}\{\mathbf{B X}\}+\sigma_{d}^{2}} \\
\text { s.t. } & X_{i i} \leq q_{i} \forall i=1, \ldots, N \\
& \mathbf{1}_{N}^{T}|\mathbf{X}| \mathbf{1}_{N} \leq \gamma ; \quad \mathbf{X} \succeq 0 \\
& \operatorname{rank}(\mathbf{X})=1,
\end{array}
$$

where $q_{i}$ is the $i$ th component of the vector $\mathbf{q}$ defined as $\mathbf{q}=\left[P_{1} / D_{11}, \ldots, P_{N} / D_{N N}\right]^{T}$. By dropping the rank-one constraint the following problem is obtained

$$
\begin{array}{cl}
\max _{\mathbf{X}} & \frac{\operatorname{Tr}\{\mathbf{A X}\}}{\operatorname{Tr}\{\mathbf{B X}\}+\sigma_{d}^{2}} \\
\text { s.t. } & X_{i i} \leq q_{i} \quad \forall i=1, \ldots, N \\
& \mathbf{1}_{N}^{T}|\mathbf{X}| \mathbf{1}_{N} \leq \gamma ; \quad \mathbf{X} \succeq 0 .
\end{array}
$$

Unfortunately, the semidefinite relaxation does not immediately yield a semidefinite programming. Due to the fractional structure of its objective (21a), this problem is a quasi-convex problem in the variable $\mathbf{X}$. The standard approach for solving this type of problems in the signal processing literature is to use a bisection search method [3], [24] in which the solution is sequentially searched by solving a sequence of (often many) semidefinite programming problems. A different approach has been considered herein. The main idea is to 
reformulate the quasi-convex problem presented in (21) into a convex semidefinite problem using a slight modification of the conventional Charnes-Cooper transformation [25]. Consider the following transformation of variables:

$$
z=\frac{1}{\operatorname{Tr}\{\mathbf{B X}\}+\sigma_{d}^{2}}, \quad \mathbf{Y}=\frac{\mathbf{X}}{\operatorname{Tr}\{\mathbf{B X}\}+\sigma_{d}^{2}}=z \mathbf{X} .
$$

By using (22), the quasi-convex problem (21) can be rewritten as the following semidefinite program (SDP)

$$
\begin{array}{ll}
\max _{\mathbf{Y}, z} & \operatorname{Tr}\{\mathbf{A Y}\} \\
\text { s.t. } & Y_{i i} \leq z q_{i} \quad \forall i=1, \ldots, N \\
& \mathbf{1}_{N}^{T}|\mathbf{Y}| \mathbf{1}_{N} \leq z \gamma \\
& \operatorname{Tr}\{\mathbf{B Y}\}+\sigma_{d}^{2} z=1 \\
& \mathbf{Y} \succeq 0 ; \quad z \geq 0,
\end{array}
$$

with $z \in \mathbb{R}$ and $\mathbf{Y} \in H_{N}^{+}=\left\{\mathbf{Y} \in \mathbb{C}^{N \times N} \mid \mathbf{Y}=\mathbf{Y}^{H}, \mathbf{Y} \succeq 0\right\}$, under the assumption that the optimal solution, denoted by $\left(\mathbf{Y}^{*}, z^{*}\right)$, has $z^{*}>0$. Actually, $z^{*}=0$ cannot be a solution of this problem, because if $z^{*}=0$, then according to $(23 \mathrm{c})$, we have $\mathbf{Y}^{*}=\mathbf{0}$, which violates the constraint (23d). This proves the equivalence between the quasi-convex problem in (21) and the SDP presented in (23). Hence, if $\left(\mathbf{Y}^{*}, z^{*}\right)$ is the optimal solution of (23), then $\mathbf{X}^{*}=\mathbf{Y}^{*} / z^{*}$ is the optimal solution of (21). The problem presented in (23) can be solved using the standard interior point methods implemented in solvers such as SeDuMi [26].

To determine the subset of selected nodes the following procedure is considered. The non-zero elements of diagonal $\mathbf{Y}^{*}$ correspond the selected relays. On the contrary, the null diagonal entries correspond to the relays that should be left out of the transmission. Note that since the active elements of the diagonal of $\mathbf{Y}^{*}$ are the same than those of the matrix $\mathbf{X}^{*}$, then the change of variables does not need to be undone.

\section{B. Computation of the network beamforming weights}

Once the subset of $K$ relays is selected, the network beamforming weights which maximize the SNR have to be computed. Due to the influence of the $l_{1}$-norm squared behind the constraint (23c), these weights cannot be directly extracted from the solution of (23). To compute the beamformer weights this constraint and the subset of inactive relays have to be removed from this problem. Let us denote by $S \subseteq\{1, \ldots, N\}$ the subset of $K$ nodes selected for the retransmission and by $\tilde{\mathbf{w}}=\left[w_{S(1)}, \ldots, w_{S(K)}\right]^{T}$ the weights of the active relays. To find the coefficients of the optimal beamforming, the following reduced-size problem has to be solved:

$$
\begin{aligned}
\max _{\tilde{\mathbf{Y}}, z} & \operatorname{Tr}\{\tilde{\mathbf{A}} \tilde{\mathbf{Y}}\} \\
\text { s.t. } & \tilde{Y}_{i i} \leq z \tilde{q}_{i} \quad \forall i=1, \ldots, K \\
& \operatorname{Tr}\{\tilde{\mathbf{B}} \tilde{\mathbf{Y}}\}+\sigma_{d}^{2} z=1 \\
& \tilde{\mathbf{Y}} \succeq 0 \\
& z \geq 0,
\end{aligned}
$$

where $\tilde{\mathbf{A}}$ and $\tilde{\mathbf{B}}$ are the submatrices of $\mathbf{A}$ and $\mathbf{B}$ formed by selecting the rows and columns which correspond to the active relays. Note that $\tilde{\mathbf{B}}$ and $\tilde{\mathbf{A}}$ preserve the special structure of the original matrices, i.e., $\tilde{\mathbf{B}}$ is a diagonal matrix and $\tilde{\mathbf{A}}$ is DPR1 which can be decomposed as $\tilde{\mathbf{A}}=\tilde{\boldsymbol{\Lambda}}+\tilde{\mathbf{v}} \tilde{\mathbf{v}}^{H}$, with obvious definitions of $\tilde{\boldsymbol{\Lambda}}$ and $\tilde{\mathbf{v}}$. In the same way, $\tilde{q}_{i}$ denotes the $i$ th entry of the vector $\tilde{\mathbf{q}}$ which is obtained by removing the inactive relays from the vector $\mathbf{q}$. Note that $\tilde{\mathbf{Y}}$ is a square matrix of size $K$ formed by the active rows and columns of Y.

Theorem 1: At least one of the inequalities in (24b) has to be fulfilled with equality.

Proof: See Appendix A.

Due to the semidefinite relaxation, the solution of the problem described above may not be rank-one in general. Interestingly, it can be proved that: i) when $\tilde{\mathbf{A}}$ is a diagonal matrix the solution of the problem (24) can be computed exactly by means of a linear programming problem (LP); ii) when the matrix $\tilde{\mathbf{A}}$ is not diagonal the beamformer weights can be extracted from the diagonal elements of the solution. Notice that no eigendecomposition or randomization is needed to extract the coefficients. In what follows both cases are analyzed.

When the matrix $\tilde{\mathbf{A}}$ is diagonal, it is straightforward to rewrite the problem in (24) as the following linear programming problem

$$
\begin{array}{cl}
\max _{\mathbf{u} \in \mathbb{R}^{K}, z} & \mathbf{a}^{T} \mathbf{u} \\
\text { s.t. } & u_{i} \leq z \tilde{q}_{i} \quad \forall i=1, \ldots, K \\
& \mathbf{b}^{T} \mathbf{u}+\sigma_{d}^{2} z=1 \\
& u_{i} \geq 0 \quad \forall i=1, \ldots, K ; \quad z \geq 0,
\end{array}
$$

where vectors $\mathbf{a}$ and $\mathbf{b}$ contain the diagonal entries of the matrices $\tilde{\mathbf{A}}$ and $\tilde{\mathbf{B}}$ respectively, i. e., $\mathbf{a}=\left[\tilde{A}_{11}, \ldots, \tilde{A}_{K K}\right]^{T}$, $\mathbf{b}=\left[\tilde{B}_{11}, \ldots, \tilde{B}_{K K}\right]^{T}$ and the $i$ th entry of the vector $\mathbf{u}$ is given by $u_{i}=z\left|w_{S(i)}\right|^{2}$. Note that when $\tilde{\mathbf{A}}$ is a diagonal matrix, the maximization of the SNR does not depend on the phase of the beamforming vector. Thus, the optimal beamformer can be directly obtained as $\tilde{\mathbf{w}}=$ $\left[\sqrt{u_{1} / z}, \ldots, \sqrt{u_{K} / z}\right]^{T}$.

If the matrix $\tilde{\mathbf{A}}$ is not diagonal but fulfills the condition exposed in the following theorem, the solution of the problem exposed in (24) always has rank one.

Theorem 2: If the matrix $\tilde{\mathbf{A}}$ is not diagonal, but all the elements of the vector $\tilde{\mathbf{v}}$ are different from zero and $\left(\tilde{\mathbf{Y}}^{*}, z^{*}\right)$ is the solution of the problem in (24), then $\tilde{\mathbf{Y}}^{*}$ has rank one.

Proof: See Appendix B.

If the conditions exposed in the last theorem are fulfilled, $\tilde{\mathbf{w}}$ can be obtained directly from the eigendecomposition of the rank-one matrix $\mathbf{Q}=\tilde{\mathbf{Y}}^{*} / z^{*}$. Nonetheless, the computational cost of the eigendecomposition can be avoided as is described next. Since the phase of the complex weights can be obtained from $\tilde{\mathbf{v}}$ by considering $\angle \tilde{w}_{i}=\angle \tilde{v}_{i}$, where $\tilde{w}_{i}$ and $\tilde{v}_{i}$ denote the $i$ th entries of the vectors $\tilde{\mathbf{w}}$ and $\tilde{\mathbf{v}}$, respectively, then it only remains to compute their moduli. These can be obtained 
from the elements of the diagonal of the matrix $\mathbf{Q}$. Therefore, it is straightforward showing that the $i$ th entry of $\mathbf{w}_{S}$ is given by

$$
\tilde{w}_{i}=\sqrt{\frac{\tilde{Y}_{i i}^{*}}{z^{*}}} e^{j \angle \tilde{v}_{i}} \quad \forall i=1, \ldots, K,
$$

If some of the entries of $\tilde{\mathbf{v}}$ are zero, the optimal weights can be obtained in a similar way. This is justified by the following theorem.

Theorem 3: If some of the elements of $\tilde{\mathbf{v}}$ are zero and $\left(\tilde{\mathbf{Y}}^{*}, z^{*}\right)$ is the solution of (24), then the ith entry of $\tilde{\mathbf{w}}$ is given by:

$$
\tilde{w}_{i}= \begin{cases}\sqrt{\frac{\tilde{Y}_{i i}^{*}}{z^{*}}} & \text { if } \quad \tilde{v}_{i}=0 \\ \sqrt{\frac{\tilde{Y}_{i i}^{*}}{z^{*}}} e^{j \angle \tilde{v}_{i}} & \text { if } \quad \tilde{v}_{i} \neq 0 .\end{cases}
$$

Proof: See Appendix C.

This result can be seen as a generalization of (26) if one assume that $\angle \tilde{v}_{i}=0$ when $\tilde{v}_{i}=0$. Notice that even if $\tilde{\mathbf{A}}$ is not diagonal the entries of the vector can be directly obtained from the solution of (24) and no randomization or eigendecomposition is needed.

A direct consequence of the Theorems 1-3 is that at least one of the relays has to transmit with the maximum allowable power.

\section{Parameter selection}

A crucial part of the algorithm is the proper choice of the parameter $\gamma$ in (23c) because it performs the selection of the subset of relays. This parameter controls the amount of shrinkage applied to the estimates and, consequently, the number of the active nodes in the optimal beamformer. It is worth noting that sparser solutions are obtained when $\gamma$ is decreased. The goal of this subsection is to propose a method based on a binary search over the parameter $\gamma$ that successively increase the sparsity of the vector $\mathbf{w}$ until the desired number of relays is selected.

Recall the inequality in (18c), i.e., $\|\mathbf{w}\|_{1}^{2} \leq \gamma$ and consider the following useful bounds on the $l_{1}$-norm squared [27]

$$
\|\mathbf{w}\|_{2}^{2} \leq\|\mathbf{w}\|_{1}^{2} \leq K\|\mathbf{w}\|_{2}^{2} .
$$

This last expression connects the $l_{1}$-norm squared with the $l_{2}$-norm and the desired cardinality of the vector $\mathbf{w}$, which is $K$. First of all, we need to determine an initial value of the parameter $\gamma$ in the binary search procedure. This value, which is denoted by $\gamma_{\max }$, has to ensure that the obtained solution will have, at least, $K$ active relays. With this aim in mind let us focus on the right side of the inequality, i.e., $\|\mathbf{w}\|_{1}^{2} \leq K\|\mathbf{w}\|_{2}^{2}$. If an upper bound on $l_{2}$-norm squared of w can be determined, it can be used to compute $\gamma_{\max }$. To obtain this bound consider the problem in (24) assuming that all the relays are active, i.e., consider $\tilde{\mathbf{A}}=\mathbf{A}$ and $\tilde{\mathbf{B}}=\mathbf{B}$ and $\tilde{\mathbf{q}}=\mathbf{q}$ and let $\mathbf{w}^{(0)}$ be the optimal beamformer obtained from the solution of this problem. From (18c) and (28), it is clear that $\gamma=K\left\|\mathbf{w}^{(0)}\right\|_{2}^{2}$ ensures that at least $K$ relays will be active. This is due to the fact that by decreasing $\gamma$ one is also decreasing $\|\mathbf{w}\|_{1}^{2}$ and, consequently, $\|\mathbf{w}\|_{2}^{2}$. Thus, $\gamma_{\max }=$ $K\left\|\mathbf{w}^{(0)}\right\|_{2}^{2}$ will be used as initial value in the search process. Unfortunately, $\gamma=\gamma_{\max }$ often enforces solutions with more than $K$ active entries in the solution vector. Therefore, we need to decrease the parameter $\gamma$ by considering a binary search until a solution with the desired number of active relays is obtained. The whole algorithm is summarized in the following subsection. Note that this binary search requires solving the problem in (23) for different values of $\gamma$ until a solution with the desired degree of sparsity is obtained. Nevertheless, the number of semidefinite programming problems which needs to be solved with this binary search is far less than in the exhaustive search which requires solving $\left(\begin{array}{c}N \\ K\end{array}\right)$ problems of type (24). This fact will be further analyzed in Section IV.

\section{Description of the algorithm}

The whole method is summarized in Algorithm 1.

\begin{tabular}{l}
\hline Algorithm 1 Proposed method \\
\hline STEP 1) INITIALIZATION: Solve (24) assuming that all the relays \\
are active and obtain $\mathbf{w}^{(0)}$. Initialize the values for the binary \\
search: $\gamma_{\max }=K\left\|\mathbf{w}^{(0)}\right\|_{2}^{2}, \gamma_{\text {low }}=0, \gamma=\gamma_{\max }$.
\end{tabular}

STEP 2) SELECTION OF THE SUBSET OF RELAYS: while number of active relays $\neq K$ do

A) Solve (23) for the corresponding $\gamma$ and determine the active relays (non-zero entries of the diagonal of $\mathbf{Y}$ )

B) Compute the new value of $\gamma$ as follows

if number of active relays $>K$ then $\gamma_{u p}=\gamma$ and $\gamma \leftarrow\left(\gamma_{l o w}+\gamma\right) / 2$

else

if number of active relays $<K$ then

$\gamma_{\text {low }}=\gamma$ and $\gamma \leftarrow\left(\gamma_{u p}+\gamma\right) / 2$

end if

end while

STEP 3) COMPUTATION OF THE WEIGHTS: Solve the reduced-size problem (24) with the selected subset and extract the weights $\tilde{\mathbf{w}}$.

\section{E. Relationship between total relay transmit power and pa-} rameter $\gamma$

To analyze this relationship let us explore the expression of the total relay transmit power which is given by

$$
P_{T}=\sum_{i=1}^{i=N}\left|y_{i}\right|^{2}=\sum_{i=1}^{i=N} E\left\{\left|x_{i}\right|^{2}\right\}\left|w_{i}\right|^{2}=\mathbf{w}^{H} \mathbf{D} \mathbf{w},
$$

where $y_{i}$ and the diagonal matrix $\mathbf{D}$ have been defined in (2) and (13) respectively. Applying the Cauchy-Schwarz inequality

$$
P_{T}=\mathbf{w}^{H} \mathbf{D} \mathbf{w}=\left\|\mathbf{D}^{1 / 2} \mathbf{w}\right\|_{2}^{2} \leq\left\|\mathbf{D}^{1 / 2}\right\|_{2}^{2}\|\mathbf{w}\|_{2}^{2} .
$$


Bearing in mind (30), the left side inequality in (28) and (18c), it is straightforward to show

$$
P_{T} \leq\left\|\mathbf{D}^{1 / 2}\right\|_{2}^{2}\|\mathbf{w}\|_{2}^{2} \leq\left\|\mathbf{D}^{1 / 2}\right\|_{2}^{2}\|\mathbf{w}\|_{1}^{2} \leq\left\|\mathbf{D}^{1 / 2}\right\|_{2}^{2} \gamma
$$

From this inequality one can conclude that if the parameter $\gamma$ is shrunk to promote the desired degree of sparsity, the power transmitted by the relays $P_{T}$ is also decreased.

\section{Numerical Results}

The goal of this section is to analyze the performance of the algorithm exposed above by numerical simulations. To solve the semidefinite problems presented in (23) and (24), CVX [28], a MATLAB package for disciplined convex programming, is used. Next, we describe the set of parameters considered throughout the simulations.

The first scenario under consideration is a wireless network composed of a source, which transmits with a power $P_{s}=$ $0 \mathrm{dBW}$, a destination and $N=20$ potential relays whose individual power constraints are uniformly given by

$$
P_{i}=\frac{P}{N} \quad \text { (in W) } \quad \text { for } i=1, \ldots, N .
$$

with $P=20 \mathrm{~W}$ (which implies $P_{i}=0 \mathrm{dBW}$ ). The noise variances are set to $\sigma_{d}^{2}=\sigma_{r}^{2}=-3 \mathrm{dBW}$.

Figure 2 plots the achieved SNR as a function of the number of selected relays $K$. The curves were obtained by averaging the results of 1000 independent simulation runs. In each trial, the means and the variances of the flat fading channels were generated randomly as follows:

$$
\begin{aligned}
& \bar{h}_{i}, \bar{g}_{i} \sim \mathcal{C N}(0,1) \text { for } i=1, \ldots, N \\
& \alpha_{i}, \beta_{i} \sim \frac{1}{2} \mathcal{X}^{2}(2) \text { for } i=1, \ldots, N
\end{aligned}
$$

where $\mathcal{X}^{2}(2)$ denotes the chi-square distribution with two degrees of freedom. The parameters $\bar{h}_{i}, \alpha_{i}, \bar{g}_{i}$ and $\beta_{i}$ denote the mean and the variance of the $i$-th element of $\mathbf{h}$ and $\mathbf{g}$, respectively, as has been exposed in (7). In each trial, the matrices $\mathbf{A}, \mathbf{B}$ and $\mathbf{D}$ have been generated according to the values of the paramaters presented above. In particular, the matrix $\mathbf{A}$ is generated following the expression (7) and $\mathbf{B}$ is created according to (10), with $E\left\{\left|g_{i}\right|^{2}\right\}=\left|\bar{g}_{i}\right|^{2}+\beta_{i}$. Regarding the matrix $\mathbf{D}$, it is formed as in (13), with $E\left\{\left|h_{i}\right|^{2}\right\}=\left|\bar{h}_{i}\right|^{2}+\alpha_{i}$.

As can be seen from Figure 2, the proposed method clearly outperforms the random selection of the relays and achieves a performance in terms of end-to-end SNR that is very close to that of the exhaustive search, requiring far less computational complexity. This fact will be analyzed later.

To gain further insight into the approximation quality of the solution described in this paper, Figure 3 plots the averaged and the maximum approximation ratios as a function of the number of selected relays. The approximation ratio is a common way to measure the quality of convex approximations in combinatorial problems and is defined as the ratio between the optimal SNR, obtained by computing the exhaustive search, and the SNR obtained by the solution of the relaxed problem $\tilde{\mathbf{w}}$. Note that the approximation ratio parameter is always greater than or equal to one and is equal to one if the relaxed problem attains the same objective value as the optimal-SNR

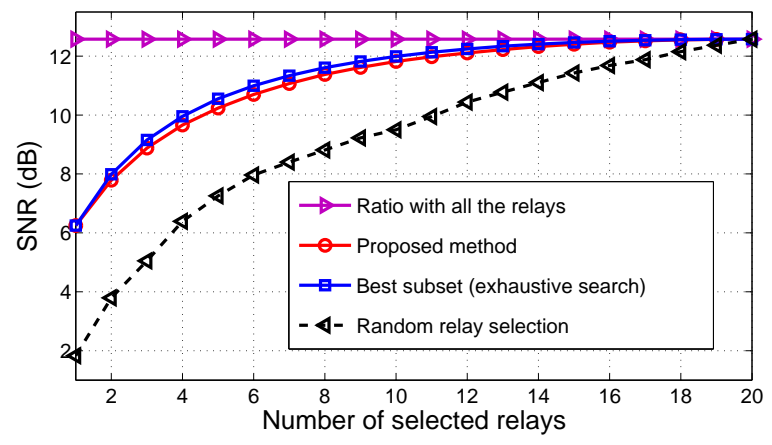

Fig. 2: SNR at the receiver as a function of the number of selected relays $K$ with uniform power constraints. $P_{i}=0 \mathrm{dBW}, \sigma_{d}^{2}=\sigma_{r}^{2}=-3 \mathrm{dBW}$. Average of the results of 1000 independent trials.

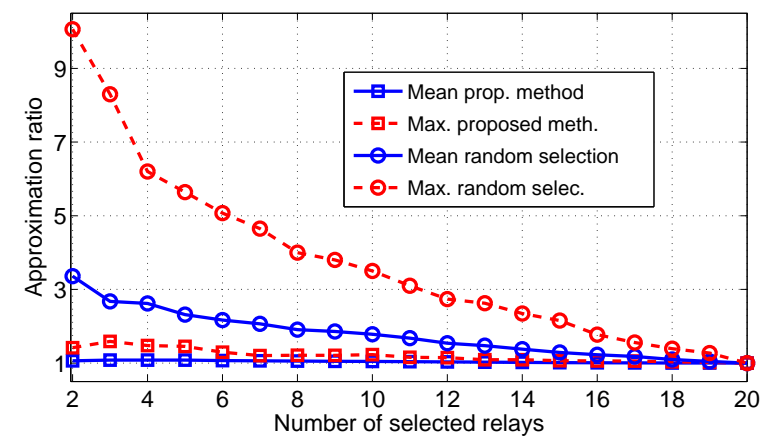

Fig. 3: Empirical approximation ratio as a function of the number of selected relays.

scheme. The proposed method clearly provides high quality approximate solutions, in terms of both the averaged and the worst case perfomance, and is close to the optimal for the all the possible cooperative sizes.

Regarding the computational complexity, Table Ia shows the mean number of semidefinite programming problems required to select the appropriate number of relays during the binary search procedure. Note that the number of iterations for each $K$ is always less than the total number of potential relays $N$. To get further insight about the reduction of the computational complexity, consider, for instance, the selection of the best subset of 12 nodes out of a potential set of 20 relays. An exhaustive search in this case requires solving 125970 SDP. Nonetheless, the proposed technique needs to solve less than 7 SDP problems in mean (less than 6 for the selection of the subset plus 1 for the computation of the optimal weights). Furthermore, the worst case required the computation of 15 SDP problems which is far less than the number of SDP needed by the exhaustive search.

In order to analyze the performance of the method for different individual power values, Figure 4 plots the achieved SNR versus the individual power constraints for different values of $K$. In this case, the value of the uniform power constraints presented in (32) is varied. Note that the proposed algorithm exhibits a good performance and is close to the optimal solution for all the possible individual power levels.

Next, we investigate the performance of the algorithm when 


\begin{tabular}{|c|c|c|}
\hline$K$ & $\begin{array}{c}\text { Mean num. it. } \\
\text { Prop. meth. }\end{array}$ & $\begin{array}{c}\text { Num. SDP } \\
\text { Exhaustive }\end{array}$ \\
\hline 2 & 6.35 & 190 \\
\hline 3 & 7.08 & 1140 \\
\hline 4 & 7.11 & 4845 \\
\hline 5 & 6.94 & 15504 \\
\hline 6 & 6.63 & 38760 \\
\hline 7 & 6.46 & 77520 \\
\hline 8 & 6.14 & 125970 \\
\hline 9 & 6.09 & 167960 \\
\hline 10 & 6.13 & 184756 \\
\hline 11 & 6.09 & 167960 \\
\hline 12 & 5.66 & 125970 \\
\hline 13 & 5.52 & 77520 \\
\hline 14 & 4.99 & 38760 \\
\hline 15 & 4.96 & 15504 \\
\hline 16 & 4.52 & 4845 \\
\hline 17 & 4.19 & 1140 \\
\hline 18 & 3.56 & 190 \\
\hline 19 & 3.56 & 20 \\
\hline
\end{tabular}

(a) $N=20$ potential relays and uniform power constraints

\begin{tabular}{|c|c|c|}
\hline$K$ & $\begin{array}{c}\text { Mean num. it. } \\
\text { prop. meth. }\end{array}$ & $\begin{array}{c}\text { Num. SDP } \\
\text { Exhaustive }\end{array}$ \\
\hline 2 & 5.27 & 45 \\
\hline 3 & 5.40 & 120 \\
\hline 4 & 5.15 & 210 \\
\hline 5 & 4.96 & 252 \\
\hline 6 & 4.65 & 210 \\
\hline 7 & 4.51 & 120 \\
\hline 8 & 4.12 & 45 \\
\hline 9 & 3.67 & 10 \\
\hline
\end{tabular}

(b) $N=10$ potential relays and nonuniform power constraints

TABLE I: Mean number of SDP problems which needs to be solved during the binary process as a function of the number of relays.

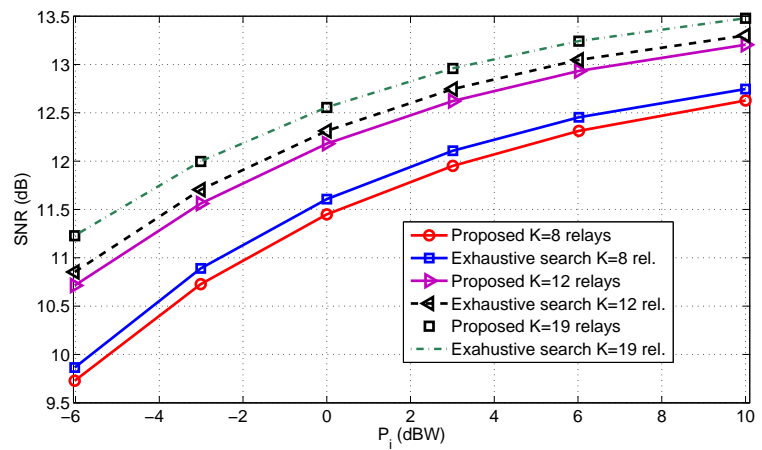

Fig. 4: SNR measured at the receiver as a function of the individual power constraints for different values of $K$

the number of potential relay nodes increases. With this aim, we have considered a wireless cooperative network composed of a source, which transmits with a power $P_{s}=0 \mathrm{dBW}$, $N$ potential relays, with the aforementioned uniform power constraints and $P=10 \mathrm{dBW}$, and the noise variances $\sigma_{d}^{2}$, $\sigma_{r}^{2}$ are set to $-3 \mathrm{dBW}$. Figure 5 shows the averaged and the maximum approximation ratio as a function of the total number of potential relays $N$ when the size of the cooperative group is fixed to $K=N / 7$. As can be seen from Figure 5, the described technique is close to the optimal solution for all the network sizes and delivers high approximate solutions in both the averaged and the worst case perfomance.

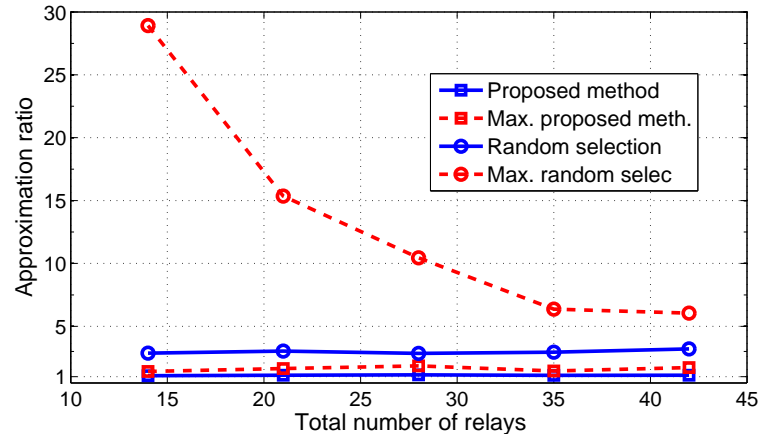

Fig. 5: Approximation ratio as a function of the total number of potential relays. $N \in[14,21,28,35,42], K=\frac{N}{7}$ and $P=10 \mathrm{dBW}$.

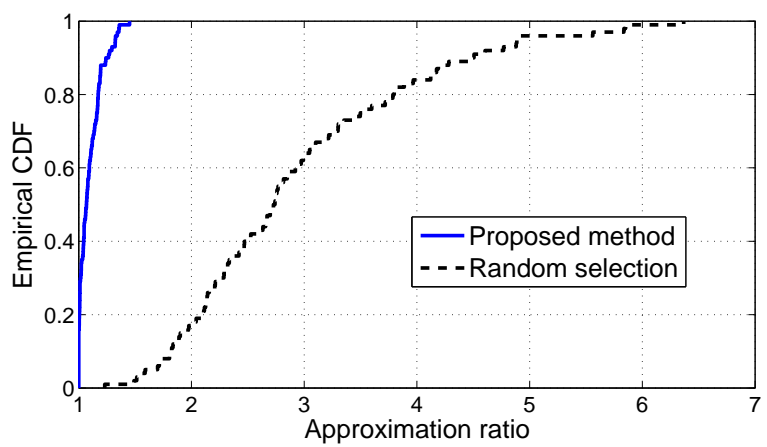

Fig. 6: Empirical cumulative distribution function of the approximation ratios. $P_{s}=0 \mathrm{dBW}, N=35, K=5$, uniform power constraints with $P=10$ $\mathrm{dBW}$ and $\sigma_{d}^{2}=\sigma_{r}^{2}=-3 \mathrm{dBW}$.

In order to illustrate the high performance of the algorithm, Figure 6 shows the empirical Cumulative Distribution Function (CDF) of the approximation ratios in the previous scenario for the case $N=35$ and $K=5$. Notice that the proposed algorithm achieves ratios that are close to the optimal value with a high probability and clearly outperforms the random selection of the subset of cooperative nodes.

Next, we consider a numerical example with non-uniform power constraints. In this case the individual constraints are set to

$$
P_{i}=i \frac{2 P}{N(N+1)} \quad(\text { in } \mathrm{W}) \quad \text { for } i=1, \ldots, N .
$$

Notice that the sum of individual power constraints is equal to $P$, as in the previous examples. For the new scenario the number of potential relays is $N=10$, the rest of parameters are set to $P_{s}=3 \mathrm{dBW}, \sigma_{d}^{2}=\sigma_{r}^{2}=0 \mathrm{dBW}$ and $P=20 \mathrm{~W}$. The results presented below are obtained by averaging the results of 1000 simulation runs and at each trial the matrices $\mathbf{A}, \mathbf{B}$ and $\mathbf{D}$ have been generated according to the procedure described above.

Figure 7 and Table Ib show the achieved SNR and the mean number of SDP problems which needs to be solved as a function of the number of selected relays, respectively. As in the uniform-constrained case, the described technique achieves a near-optimal performance with a low computational burden. Regarding the computational complexity, the worst- 


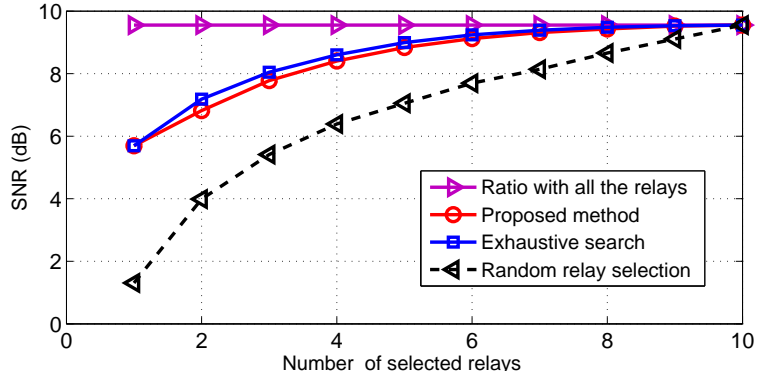

Fig. 7: SNR measured at the receiver as a function of the number of selected relays $K .10$ relays and non-uniform power constraints.

case scenario required 14 iterations and was obtained for $K=6$.

\section{CONCLUSIONS}

A new method which deals with the problem of multiple relay selection under per-relay power constraints was presented in this paper. In particular, we have addressed the joint problem of selecting the best subset of cooperative nodes and their corresponding beamforming weights so that the end-to-end SNR is maximized. The optimal solution of this problem is computationally demanding and requires an exhaustive combinatorial search. In order to reduce the computational burden, this paper has proposed a sub-optimal method with a nearoptimal performance and a feasible computational complexity. Our approach is based on the knowledge of the second-order statistics of the CSI and the relays are not limited to cooperate at full power.

\section{APPENDIX A}

\section{PROOF OF THEOREM 1}

Since the maximization problem presented in (24) is convex and feasible, the KKT conditions [24] are necessary and sufficient conditions for the optimality. Let $\left(\tilde{\mathbf{Y}}^{*}, z^{*}\right)$ be the solution to (24), then the KKT conditions of this problem are

$$
\begin{gathered}
-\tilde{\mathbf{A}}+\sum_{i=1}^{K} \lambda_{i} \mathbf{J}_{i}+\mu \tilde{\mathbf{B}}-\mathbf{\Phi}=\mathbf{0} \\
-\sum_{i=1}^{K} \lambda_{i} \tilde{q}_{i}+\mu \sigma_{d}^{2}-\delta=0 \\
\operatorname{Tr}\left\{\mathbf{J}_{i} \tilde{\mathbf{Y}}^{*}\right\}-z^{*} \tilde{q}_{i} \leq \mathbf{0} \quad \forall i=1, \ldots, K \\
\operatorname{Tr}\left\{\tilde{\mathbf{B}} \tilde{\mathbf{Y}}^{*}\right\}+\sigma_{d}^{2} z^{*}-1=0 ; \tilde{\mathbf{Y}}^{*} \succeq 0 ; z^{*} \geq 0 \\
\lambda_{i} \geq 0 \quad \forall i ; \quad \mathbf{\Phi} \succeq 0 \\
\lambda_{i}\left[\operatorname{Tr}\left\{\mathbf{J}_{i} \tilde{\mathbf{Y}}^{*}\right\}-z^{*} \tilde{q}_{i}\right]=0 \quad \forall i=1, \ldots, K \\
\operatorname{Tr}\left\{\boldsymbol{\Phi} \tilde{\mathbf{Y}}^{*}\right\}=0 \\
\delta z^{*}=0,
\end{gathered}
$$

where $\mathbf{J}_{i}$ is a single-entry matrix, with zeros in all the entries except for the $(i, i)$ th element which is equal to one. The parameters $\lambda_{i}, \mu, \boldsymbol{\Phi}$ and $\delta$ are the Lagrange multipliers associated with the constraints (24b)-(24e) respectively.

As it was discussed in the subsection III-A, $z^{*}=0$ cannot be a solution of the problem (23), and this imposes $\delta=0$ in the equation (35h). Consider that all the relays do not use their maximum power in the retransmission. Taking into account (35f), this implies $\lambda_{i}=0 \forall i$. Consequently, as $\delta=0$, $\lambda_{i}=0 \forall i$, it is clear from (35b) that $\mu=0$. Bearing in mind these values, equation (35a) can be rewritten as $\boldsymbol{\Phi}=-\tilde{\mathbf{A}}$ which does not make sense because $\boldsymbol{\Phi} \succeq 0$ and $\tilde{\mathbf{A}} \succeq 0$.

\section{APPENDIX B \\ PROOF OF THEOREM 2}

Recall the KKT conditions presented in Appendix A (35a) - (35h). Taking into account (35g), as $\boldsymbol{\Phi} \succeq 0$ and $\tilde{\mathbf{Y}}^{*} \succeq 0$, then it is clear that $\boldsymbol{\Phi} \tilde{\mathbf{Y}}^{*}=\mathbf{0}$. As the matrices $\tilde{\mathbf{Y}}^{*}$ and $\boldsymbol{\Phi}$ are square matrices of size $K \times K$, then the Sylvester's rank inequality holds (Section 10.5.1 of [29])

$$
\operatorname{rank}\left(\boldsymbol{\Phi} \tilde{\mathbf{Y}}^{*}\right) \geq \operatorname{rank}(\boldsymbol{\Phi})+\operatorname{rank}\left(\tilde{\mathbf{Y}}^{*}\right)-K
$$

As the $\operatorname{rank}\left(\boldsymbol{\Phi} \tilde{\mathbf{Y}}^{*}\right)=0$, then

$$
\operatorname{rank}\left(\tilde{\mathbf{Y}}^{*}\right) \leq K-\operatorname{rank}(\boldsymbol{\Phi}) .
$$

To determine the $\operatorname{rank}\left(\tilde{\mathbf{Y}}^{*}\right)$ we need to compute the $\operatorname{rank}(\boldsymbol{\Phi})$. With this aim let us recall (35a). As the matrix $\tilde{\mathbf{A}}$ can be expressed in terms of the sum of diagonal matrix plus a rankone matrix (7) $\tilde{\mathbf{A}}=\tilde{\boldsymbol{\Lambda}}+\tilde{\mathbf{v}} \tilde{\mathbf{v}}^{H}$. Then equation in (35a) can be rewritten as

$$
\left[\sum_{i=1}^{K} \lambda_{i} \mathbf{J}_{i}-\tilde{\boldsymbol{\Lambda}}+\mu \tilde{\mathbf{B}}\right]-\tilde{\mathbf{v}} \tilde{\mathbf{v}}^{H}=\boldsymbol{\Phi}
$$

Note that the matrix $\sum_{i=1}^{K} \lambda_{i} \mathbf{J}_{i}-\tilde{\mathbf{\Lambda}}+\mu \tilde{\mathbf{B}}$ is a diagonal matrix. Now let us prove that the entries of this matrix are all greater than zero. To prove that fact, consider that the $i$ th element of diagonal of the matrix $\sum_{i=1}^{K} \lambda_{i} \mathbf{J}_{i}-\tilde{\boldsymbol{\Lambda}}+\mu \tilde{\mathbf{B}}$ is less than or equal to zero and consider a single-entry vector $\mathbf{s}_{i}$ with all the elements equal to zero except for the $i$ th element which is equal to one. If all the entries of the vector $\tilde{\mathbf{v}}$ are different from zero, then $\mathbf{s}_{i}^{H} \tilde{\mathbf{v}} \tilde{\mathbf{v}}^{H} \mathbf{s}_{i}>0$ and consequently,

$$
\mathbf{s}_{i}^{H} \mathbf{\Phi} \mathbf{s}_{i}=\mathbf{s}_{i}^{H}\left[\sum_{i=1}^{K} \lambda_{i} \mathbf{J}_{i}-\tilde{\boldsymbol{\Lambda}}+\mu \tilde{\mathbf{B}}\right] \mathbf{s}_{i}-\mathbf{s}_{i}^{H} \tilde{\mathbf{v}} \tilde{\mathbf{v}}^{H} \mathbf{s}_{i}<0 .
$$

However, this violates $\boldsymbol{\Phi} \succeq 0$. Therefore, $\boldsymbol{\Psi}=\sum_{i=1}^{K} \lambda_{i} \mathbf{J}_{i}-\tilde{\boldsymbol{\Lambda}}+$ $\mu \tilde{\mathbf{B}} \succ 0$ because $\boldsymbol{\Psi}$ is a diagonal matrix with positive entries. Furthermore, it follows from this statement that

$$
\operatorname{rank}(\boldsymbol{\Psi})=K
$$

This result will be important in the computation of $\operatorname{rank}(\boldsymbol{\Phi})$. At this point let us recall the expression (38). Using 
the following result in matrix theory [29] $\operatorname{rank}(\mathbf{U}+\mathbf{V}) \leq$ $\operatorname{rank}(\mathbf{U})+\operatorname{rank}(\mathbf{V})$, it is straightforward to rewrite $\operatorname{rank}(\mathbf{\Phi})$ in terms of the next inequality

$$
\operatorname{rank}(\boldsymbol{\Phi}) \geq \operatorname{rank}(\boldsymbol{\Psi})-\operatorname{rank}\left(\tilde{\mathbf{v}} \tilde{\mathbf{v}}^{H}\right)=K-1
$$

Thus, from the expressions (37) and (41) it follows that

$$
\operatorname{rank}\left(\tilde{\mathbf{Y}}^{*}\right) \leq K-\operatorname{rank}(\boldsymbol{\Phi}) \leq K-K+1 .
$$

Therefore, since $\tilde{\mathbf{Y}}^{*}=\mathbf{0}$ cannot maximize the objective function (24), we can conclude that $\operatorname{rank}\left(\tilde{\mathbf{Y}}^{*}\right)=1$.

\section{APPENDIX C \\ PROOF OF THEOREM 3}

If some of the elements of $\tilde{\mathbf{v}}$ are zero, the SDP problem in (24) can have a solution $\left(\tilde{\mathbf{Y}}^{*}, z^{*}\right)$ where the rank of $\tilde{\mathbf{Y}}^{*}$ is greater than one. Nevertheless, if the solution has a rank greater than one, the rank-one solution can be recovered as it is proved in this Appendix.

Denote by $J$ the set of inactive components of $\tilde{\mathbf{v}}$, i. e., $J=\left\{l \mid \tilde{v}_{l}=0\right\}$ and let $\left(\tilde{\mathbf{Y}}^{*}, z^{*}\right)$ be the solution of (24). Consider a matrix $\mathbf{C}$ defined as

$$
C_{l k}= \begin{cases}\tilde{Y}_{l k}^{*} & \text { if } l=k \text { or } l, k \notin J \\ 0 & \text { otherwise, }\end{cases}
$$

where $C_{l k}$ denotes the $(l, k)$ th element of the matrix C. It can be proved that $\left(\mathbf{C}, z^{*}\right)$ is also a feasible point of (24) that achieves the same objective value as $\left(\tilde{\mathbf{Y}}^{*}, z^{*}\right)$. To prove this, first of all, let us decompose $\mathbf{C}$ as follows:

$$
\mathbf{C}=\mathbf{E}+\mathbf{F},
$$

where $\mathbf{E}$ is a sparse matrix whose active components are the entries of the diagonal corresponding to the elements of the set $J$

$$
E_{l l}= \begin{cases}\tilde{Y}_{l l}^{*} & \text { if } l \in J \\ 0 & \text { otherwise }\end{cases}
$$

and $\mathbf{F}$ a matrix whose $(l, k)$ th entry is given by

$$
F_{l k}= \begin{cases}\tilde{Y}_{l k}^{*} & \text { if } l \notin J, k \notin J \\ 0 & \text { otherwise. }\end{cases}
$$

Recall that the aim of this appendix is to prove that even if $\tilde{\mathbf{Y}}^{*}$ has a rank greater than one, the rank-one solution can be recovered directly from $\tilde{\mathbf{Y}}^{*}$ and $z^{*}$ and no randomization or eigendecomposition is needed. The following lemma is the key point of the proof.

Lemma 1: If $\left(\tilde{\mathbf{Y}}^{*}, z^{*}\right)$ is the solution of the SDP in (24), then $\left(\mathbf{C}, z^{*}\right)$ is a feasible point which achieves the same objective value as $\left(\tilde{\mathbf{Y}}^{*}, z^{*}\right)$. Furthermore, $\mathbf{C}$ can be decomposed as $\mathbf{C}=\mathbf{E}+\mathbf{F}$, where $\mathbf{E}$ and $\mathbf{F}$ the matrices exposed above, and $\mathbf{F}$ is a rank-one matrix.

\section{Proof: See Appendix D.}

The proof of the first part of the lemma is based on fact that $\mathbf{C}$ differs from $\tilde{\mathbf{Y}}^{*}$ only at the entries given by $(l, k)$ with $l, k \in J$ and $l \neq k$. Due to this fact, $\left(\mathbf{C}, z^{*}\right)$ is a feasible point which attains the same objective function. Furthermore, since $\mathbf{F}$ is a rank-one matrix, it can be decomposed as $\mathbf{F}=\mathrm{ff}^{H}$.
Consider $\mathbf{H} \triangleq \mathbf{h} \mathbf{h}^{H}$, where $\mathbf{h}$ is a vector whose $l$ th entry is given by

$$
h_{l}= \begin{cases}\sqrt{\tilde{Y}_{l l}^{*}} & \text { if } l \in J \\ f_{l} & \text { otherwise }\end{cases}
$$

where $f_{l}$ denotes the $l$ th element of $\mathbf{f}$. Following an analysis similar to the proof of Lemma1 it is straightforward showing that $\left(\mathbf{H}, z^{*}\right)$ is a feasible point that achieves the same objective value as $\left(\tilde{\mathbf{Y}}^{*}, z^{*}\right)$. The key point of this proof is that $\mathbf{H}$ differs from $\tilde{\mathbf{Y}}^{*}$ only at the entries given by $(l, k)$ with $l, k \in J$ and $l \neq k$. Bearing in mind that fact and since $\mathbf{H}$ is rank one, the optimal beamformer can be recovered from the eigendecomposition of $\mathbf{H} / z^{*}$, i. e., $\tilde{\mathbf{w}}=h / \sqrt{z^{*}}$. Thus, the optimal weights can be obtained as

$$
\tilde{w}_{l}= \begin{cases}\sqrt{\frac{\tilde{Y}_{l l}^{*}}{z^{*}}} & \text { if } \quad l \in J \\ \frac{f_{l}}{\sqrt{z^{*}}} & \text { otherwise. }\end{cases}
$$

Notice that the moduli of the diagonal entries of $\mathbf{F}$ (46) not belonging to $J$ can be directly extracted from $\tilde{\mathbf{Y}}^{*}$. As a consequence, $\left|f_{l}\right|=\sqrt{\tilde{Y}_{l l}^{*}}$ for $l \notin J$. It only remains to compute the phases and they can be obtained from the components of $\tilde{\mathbf{v}}$. Therefore, the next result is obtained:

$$
\tilde{w}_{l}= \begin{cases}\sqrt{\frac{\tilde{Y}_{l l}^{*}}{z^{*}}} & \text { if } l \in J \\ \sqrt{\frac{\tilde{Y}_{l l}^{*}}{z^{*}}} e^{j<\tilde{v}_{l}} & \text { otherwise, }\end{cases}
$$

where $\tilde{v}_{l}$ denotes the $l$ th element of $\tilde{\mathbf{v}}$. This concludes the proof of the theorem.

\section{APPENDIX D}

\section{PROOF OF LEMMA 1}

To prove the first part of the lemma, i.e., to prove that $\left(\mathbf{C}, z^{*}\right)$ is a feasible point that achieves the same objective value as $\left(\tilde{\mathbf{Y}}^{*}, z^{*}\right)$ we need to show that:

1) $\operatorname{Tr}\left\{\tilde{\mathbf{A}} \tilde{\mathbf{Y}}^{*}\right\}=\operatorname{Tr}\{\tilde{\mathbf{A}} \mathbf{C}\}$

2) $C_{i i}=\tilde{Y}_{i i}^{*} \leq z^{*} \tilde{q}_{i} \quad \forall i=1, \ldots, K$

3) $\operatorname{Tr}\left\{\tilde{\mathbf{B}} \tilde{\mathbf{Y}}^{*}\right\}+\sigma_{d}^{2} z^{*}=\operatorname{Tr}\{\tilde{\mathbf{B}} \mathbf{C}\}+\sigma_{d}^{2} z^{*}=1$

4) $\mathbf{C} \succeq 0$

Let us analyze it point by point:

1) $\operatorname{Tr}\left\{\tilde{\mathbf{A}} \tilde{\mathbf{Y}}^{*}\right\}=\operatorname{Tr}\{\tilde{\mathbf{A}} \mathbf{C}\}$ implies $\operatorname{Tr}\left\{\tilde{\mathbf{A}}\left(\tilde{\mathbf{Y}}^{*}-\mathbf{C}\right)\right\}=0$. Considering (7), this can be rewritten as follows $\operatorname{Tr}\left\{\left(\tilde{\mathbf{\Lambda}}+\tilde{\mathbf{v}} \tilde{\mathbf{v}}^{H}\right)\left(\tilde{\mathbf{Y}}^{*}-\mathbf{C}\right)\right\}=\operatorname{Tr}\left\{\left(\tilde{\mathbf{\Lambda}}\left(\tilde{\mathbf{Y}}^{*}-\mathbf{C}\right)\right\}+\right.$ $\operatorname{Tr}\left\{\tilde{\mathbf{v}} \tilde{\mathbf{v}}^{H}\left(\tilde{\mathbf{Y}}^{*}-\mathbf{C}\right)\right\}=0$. The matrix $\tilde{\mathbf{Y}}^{*}-\mathbf{C}$ has all null entries except for the elements $(l, k)$ th and $(k, l)$ th $\forall k, l \in J$ with $k \neq l$. And it is straightforward to show that $\operatorname{Tr}\left\{\left(\tilde{\mathbf{\Lambda}}\left(\tilde{\mathbf{Y}}^{*}-\mathbf{C}\right)\right\}=0\right.$ and $\operatorname{Tr}\left\{\tilde{\mathbf{v}} \tilde{\mathbf{v}}^{H}\left(\tilde{\mathbf{Y}}^{*}-\mathbf{C}\right)\right\}$ $=0$.

2) The diagonals of the matrices $\tilde{\mathbf{Y}}^{*}$ and $\mathbf{C}$ have the same elements and then $C_{i i}=\tilde{Y}_{i i}^{*}$, then it is straightforward to show the inequality.

3) $\tilde{\mathbf{B}}$ is diagonal and the diagonals of $\tilde{\mathbf{Y}}^{*}$ and $\mathbf{C}$ have the same elements. Thus, $\operatorname{Tr}\left\{\tilde{\mathbf{B}} \tilde{\mathbf{Y}}^{*}\right\}=\operatorname{Tr}\{\tilde{\mathbf{B}} \mathbf{C}\}$, and then this point is proved.

4) Recall (44), $\mathbf{C}=\mathbf{E}+\mathbf{F}$. Since $\mathbf{E}$ is a diagonal matrix and the elements of the diagonal are zero or positive, 
then $\mathbf{E} \succeq 0$. Furthermore, $\mathbf{F}$ is obtained by putting to zero the rows and the columns of $\tilde{\mathbf{Y}}^{*}$ which correspond to the inactive components of $\tilde{\mathbf{v}}$. Since all the principal minors of the semidefinite matrix $\tilde{\mathbf{Y}}^{*}$ are also semidefinite. Then, $\mathbf{F} \succeq 0$ and as the sum of semidefinite matrices is also semidefinite, we obtain $\mathbf{C}=\mathbf{E}+\mathbf{F} \succeq 0$.

Once it has been proved that $\left(\mathbf{C}, z^{*}\right)$ is feasible solution, we need to prove that $\mathbf{F}$ is a rank-one matrix. With this aim in mind, let us define the following variables:

- $\breve{\mathbf{F}}$ is the matrix constructed by deleting the $l$ th row and the $l$ th column of $\mathbf{F} \forall l \in J$.

- $\breve{\Lambda}$ is the diagonal matrix formed by deleting the $l$ th row and the $l$ th column of $\tilde{\boldsymbol{\Lambda}} \forall l \in J$.

- $\breve{\mathbf{B}}$ is the diagonal matrix formed by deleting the $l$ th row and the $l$ th column of $\tilde{\mathbf{B}} \forall l \in J$.

- $\breve{\mathbf{v}}$ is the vector constructed by deleting $l$ th element of $\tilde{\mathbf{v}}$ $\forall l \in J$.

Notice that $\breve{\mathbf{F}}, \breve{\mathbf{\Lambda}}$ and $\breve{\mathbf{B}}$ are square matrices of size $K-|J|$ and $\mathbf{v}_{1}$ is a vector of length $K-|J|$. It can be shown that $\mathbf{F}$ is rank one because $\breve{\mathbf{F}}$ is rank one. With this end in mind, let us rewrite the objective function presented in (24a) in terms of $\breve{\mathbf{F}}$ and $\breve{\mathbf{v}}$. Using the point 1 exposed above and the equality in (44), the objective function can be rewritten as

$$
\begin{aligned}
\operatorname{Tr}\left\{\tilde{\mathbf{A}} \tilde{\mathbf{Y}}^{*}\right\} & =\operatorname{Tr}\{\tilde{\mathbf{A}} \mathbf{C}\}=\operatorname{Tr}\{\tilde{\mathbf{A}}(\mathbf{E}+\mathbf{F})\}=\operatorname{Tr}\{\tilde{\mathbf{A}} \mathbf{E}\}+\operatorname{Tr}\{\tilde{\mathbf{A}} \mathbf{F}\} \\
& =\operatorname{Tr}\{\tilde{\mathbf{A}} \mathbf{E}\}+\operatorname{Tr}\left\{\left(\breve{\mathbf{\Lambda}}+\breve{\mathbf{v}} \breve{\mathbf{v}}^{H}\right) \breve{\mathbf{F}}\right\} .
\end{aligned}
$$

The second line in (50) follows from the fact the matrix $\mathbf{F}$ has zeros in the rows and the columns corresponding to the elements of the set $J$. As a consequence, it is straightforward to show that $\operatorname{Tr}\{\tilde{\mathbf{A}} \mathbf{F}\}=\operatorname{Tr}\left\{\left(\breve{\mathbf{\Lambda}}+\breve{\mathbf{v}} \breve{\mathbf{v}}^{H}\right) \mathbf{F}\right\}=\operatorname{Tr}\left\{\left(\breve{\boldsymbol{\Lambda}}+\breve{\mathbf{v}} \breve{\mathbf{v}}^{H}\right) \breve{\mathbf{F}}\right\}$. In the same way, the constraint $(24 \mathrm{c})$ can be rewritten in terms of $\breve{\mathbf{F}}$. Consider the point 3 exposed above, then it is straightforward to show that

$$
\begin{aligned}
\operatorname{Tr}\left\{\tilde{\mathbf{B}} \tilde{\mathbf{Y}}^{*}\right\}+\sigma_{d}^{2} z^{*} & =\operatorname{Tr}\{\tilde{\mathbf{B}} \mathbf{C}\}+\sigma_{d}^{2} z^{*} \\
& =\operatorname{Tr}\{\tilde{\mathbf{B}} \mathbf{E}\}+\operatorname{Tr}\{\breve{\mathbf{B}} \breve{\mathbf{F}}\}+\sigma_{d}^{2} z^{*} .
\end{aligned}
$$

Thus, the constraint (24c) can be expressed as

$$
\operatorname{Tr}\{\tilde{\mathbf{B}} \mathbf{E}\}+\operatorname{Tr}\{\breve{\mathbf{B}} \breve{\mathbf{F}}\}+\sigma_{d}^{2} z^{*}=1 .
$$

Based on the analysis above and since $\left(\mathbf{C}, z^{*}\right)$, with $\mathbf{C}=\mathbf{E}+\mathbf{F}$, is a feasible solution of (24), then $\breve{\mathbf{F}}$ can be obtained as the solution of the next problem

$$
\begin{array}{cc} 
& \max _{\breve{\mathbf{F}}} \quad \operatorname{Tr}\{\tilde{\mathbf{A}} \mathbf{E}\}+\operatorname{Tr}\left\{\left(\breve{\mathbf{\Lambda}}+\breve{\mathbf{v}} \breve{\mathbf{v}}^{H}\right) \breve{\mathbf{F}}\right\} \\
\text { s.t. } \quad & \breve{F}_{i i}+E_{\Theta(i) \Theta(i)} \leq z^{*} \tilde{q}_{i} \quad \forall i \in 1, \ldots, K-|J| \\
& \operatorname{Tr}\{\tilde{\mathbf{B}} \mathbf{E}\}+\operatorname{Tr}\{\breve{\mathbf{B}} \breve{\mathbf{F}}\}+\sigma_{d}^{2} z^{*}=1 \\
\breve{\mathbf{F}} \succeq 0,
\end{array}
$$

where $\Theta$ denotes the set of active components, i.e., $\Theta=$ $\left\{l \mid \tilde{v}_{l} \neq 0\right\}$ and $\Theta(i)$ the $i$ th element of the set $\Theta$. Following an analysis similar to the one exposed in the proof of Theorem 2 , we can show that since all the coefficients of $\breve{\mathbf{v}}$ differ from zero, then $\breve{\mathbf{F}}$ has rank one. Since $\mathbf{F}$ can be directly reconstructed from $\breve{\mathbf{F}}$ by adding some columns and rows of zeros and adding zero rows and zero columns does not change the rank of the matrix, the desired result is obtained.

\section{ACKNOWLEDGMENT}

The authors would like to thank the anonymous reviewers for their constructive comments.

\section{REFERENCES}

[1] J. Laneman, D. Tse, and G. Wornell, "Cooperative diversity in wireless networks: efficient protocols and outage behavior," IEEE Trans. Inf. Theory, vol. 50, no. 12, pp. 3062-3080, Dec. 2004.

[2] M. Janani, A. Hedayat, T. E. Hunter, and A. Nosratinia, "Coded cooperation in wireless communications: space-time transmission and iterative decoding," IEEE Trans. Signal Processing, vol. 52, no. 2, pp. 362-371, 2004.

[3] V. Havary-Nassab, S. Shahbazpanahi, A. Grami, and Z.-Q. Luo, "Distributed beamforming for relay networks based on second-order statistics of the channel state information," IEEE Trans. Signal Processing, vol. 56, no. 9, pp. 4306-4316, Sep. 2008.

[4] Y. Zhang and R. S. Cheng, "Relay subset selection in cooperative systems with beamforming and limited feedback," IEEE Transactions on Wireless Communications, vol. 12, no. 10, pp. 5271-5281, 2013.

[5] S. Zhang and V. K. N. La, "Multi-relay selection design and analysis for multi-stream cooperative communications," IEEE Transactions on Wireless Communications, vol. 10, no. 4, pp. 173-189, 2011.

[6] H. Nguyen, H. Nguyen, and T. Le-Ngoc, "Diversity analysis of relay selection schemes for two-way wireless relay networks," Wireless Personal Communications, vol. 59, no. 2, pp. 173-189, 2011.

[7] J. Lee and N. Al-Dhahir, "Exploiting sparsity for multiple relay selection with relay gain control in large AF relay networks," IEEE Wireless Commun. Letters, vol. 2, no. 3, pp. 347-350, 2013.

[8] S. Atapattu, Y. Jing, H. Jiang, and C. Tellambura, "Relay selection schemes and performance analysis approximations for two-way networks," IEEE Transactions on Communications, vol. 61, no. 3, pp. 987 998, 2013.

[9] Y. Jing and H. Jafarkhani, "Single and multiple relay selection schemes and their achievable diversity orders," IEEE Trans. Wireless Communications, vol. 8, no. 3, pp. 1414-1423, Mar. 2009.

[10] A. S. Ibrahim, A. K. Sadek, W. Su, and K. J. R. Liu, "Cooperative communications with relay-selection: when to cooperate and whom to cooperate with?" IEEE Trans. Wireless Communications, vol. 7, no. 7, pp. 2814-2827, Jul. 2008.

[11] Y. Zhao, R. Adve, and T. J. Lim, "Symbol error rate of selection amplifyand-forward relay systems." IEEE Communications Letters, vol. 10, no. 11, pp. 757-759, 2006.

[12] D. S. Michalopoulos, G. K. Karagiannidis, T. A. Tsiftsis, and R. K. Mallik, "An optimized user selection method for cooperative diversity systems," in Proc. IEEE GLOBECOM, 2006.

[13] J. N. Laneman and G. W. Wornell, "Distributed space-time-coded protocols for exploiting cooperative diversity in wireless networks," IEEE Trans. Inf. Theory, vol. 49, no. 10, pp. 2415-2425, Oct. 2003.

[14] J. Xu, H. Zhang, D. Yuan, Q. Jin, and C.-X. Wang, "Novel multiple relay selection schemes in two-hop cognitive relay networks," in Proc. Int. Conference on Communications and Mobile Computing, pp. 307310,2011

[15] O. Mehanna, N. Sidiropoulos, and G. B. Giannakis, "Multicast beamforming with antenna selection," in Proc. of the 13th SPAWC, 2012.

[16] Y. Jing and H. Jafarkhani, "Network beamforming using relays with perfect channel information," IEEE Trans. Information Theory, vol. 55, no. 6, pp. 2499-2517, Jun. 2009.

[17] A. Piltan and S. Salari, "Distributed beamforming in cognitive relay networks with partial channel state information." IET Communications, vol. 6, no. 9, pp. 1011-1018, 2012.

[18] J. Li, A. Petropulu, and H. Poor, "Cooperative transmission for relay networks based on second-order statistics of channel state information," IEEE Trans. Signal Processing, vol. 59, no. 3, pp. 1280-1291, Mar. 2011.

[19] A. B. Gershman, N. D. Sidiropoulos, S. Shahbazpanahi, M. Bengtsson, and B. Ottersten, "Convex optimization-based beamforming: from receive to transmit and network designs," vol. 27, no. 3, pp. 62-75, 2010.

[20] F. R. Bach, R. Jenatton, J. Mairal, and G. Obozinski, "Convex optimization with sparsity-inducing norms," Optimization for Machine Learning, MIT Press, pp. 19-53, 2011.

[21] _ "Optimization with sparsity-inducing penalties," Foundations and Trends in Machine Learning, vol. 4, no. 1, pp. 1-106, 2012.

[22] R. Tibshirani, "Regression shrinkage and selection via the lasso," Journal of the Royal Statistical Society, Series B, vol. 58, pp. 267-288, 1996. 
[23] O. Mehanna, N. D. Sidiropoulos, and G. B. Giannakis, "Joint multicast beamforming and antenna selection." IEEE Transactions on Signal Processing, vol. 61, no. 10, pp. 2660-2674, 2013.

[24] S. Boyd and L. Vandenberghe, Convex Optimization. New York, NY, USA: Cambridge University Press, 2004.

[25] A. Charnes and W. W. Cooper, "Programming with linear fractional functionals," Naval Research Logistics, vol. 9, no. 3-4, pp. 181-186, 1962.

[26] J. F. Sturm, "Using sedumi 1.02, a MATLAB toolbox for optimization over symmetric cones," Optimization Methods and Software, vol. 11-12,

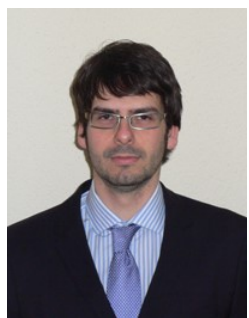

Luis Blanco received the M.S. degree in electrical engineering (Telecommunications) in 2006 and the Master's of Research on Information and Communications technologies (MERIT) in 2012, both from the Polytechnic University of Catalonia (UPC), Spain. The same year he carried out postgraduate studies in quantitative techniques for financial markets at UPC. Currently, he is finishing his Ph.D in electrical engineering at the UPC in Barcelona, Spain.

In 2006, he joined the Centre Tecnolgic de Telecomunicacions de Catalunya (CTTC), where he currently holds a position as a researcher. He received the INNOVATIA award from IDE-CESEM (Instituto de Directivos de Empresa, Madrid) in 2004. He has actively participated in several European research projects and in private funded projects with the industry. His current research interests include sparse signal processing, compressed sensing, big data and $5 \mathrm{G}$ cellular systems. pp. 625-653, 1999.

[27] N. Bourbaki, H. Eggleston, and S. Madan, Topological Vector Spaces. Springer-Verlag, 1987.

[28] "CVX: Matlab software for Disciplined Convex Programming." [Online]. Available: http://cvxr.com/cvx/

[29] K. B. Petersen and M. S. Pedersen, "The matrix cookbook," Nov. 2012. [Online]. Available: http://www2.imm.dtu.dk/pubdb/p.php?3274

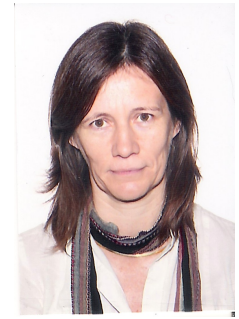

Montse Nájar (S'90-AM'96-M'08-SM'08) received the Electrical Engineering degree and the Ph.D. degree from the Polytechnic University of Catalonia (UPC), Barcelona, Spain, in 1991 and 1996, respectively. In 1992, she joined the Department of Signal Theory and Communication of the UPC. Since 1997, she has been an Associate Professor at the UPC, where she teaches and coordinates undergraduate and graduate courses in Digital Communications and Signal Processing. From 2003 to 2006, she was member of the Board of Directors of the Telecommunications School of Barcelona, ETSETB. From 2005 to 2013, she was research associate in the CTTC (Centre Tecnologic de Telecomunicacions de Catalunya). Her current research interests include signal processing with application to communication systems, array signal processing and location in wireless systems. She has participated in several IST and Eureka European projects as well as national public and private funded projects. She has been guest editor of the EURASIP Signal Processing journal. She is reviewer of the IEEE and the EURASIP Signal Processing journals. 